\title{
The Effects of Insurance on the Possibilities of Fraud
}

\author{
by Georges Dionne *
}

\section{Introduction}

According to a recent article in the New York Times (November 17, 1980), Medicare loses two billion dollars a year due to fraud. The most common type of Medicare fraud mentioned is the provision of unnecessary services to the patient. A high level of fraud was also found in the Medicaid program.

The object of this paper is to analyse the effects of insurance on the possibilities of fraud in a market characterized by a situation of asymmetrical information, between producers and consumers, concerning the technical qualities of goods or services (Darby and Karni [2], Dohan [7]). Based on the definition of Darby and Karni, fraud is the provision, by a producer, of unnecessary services to a consumer who doesn't possess full information about his or her purchase ${ }^{1}$.

This lack of information on the part of the consumer can be attributed firstly, to the cost of obtaining it which is related to the nature of the services, i.e. the joint provision of diagnoses (or estimates) and services increases the cost of search in these markets. Moreover, the consumer is often unable to fully evaluate the quality of the service offered without supplementary costs since the consumer must rely on experts to evaluate the services required. Finally, since the production function of the producers in these markets is often a stochastic function of service inputs, it is difficult for the consumer to prove that fraud has taken place. Medical and dental care, auto and T.V. repairs and other experts advice services are good examples of such markets.

In the economic literature on the effect of insurance on the resources allocation in medical care markets, for example, the focus was to explain the impact on the demand side (Arrow [1], Pauly [19], Zeckhauser [22], Feldstein [10]). In these studies it was implicitly assumed that the suppliers are perfects agents of the consumers, that is, that their actions are only related to the well-being of their patients ${ }^{2}$. In the present

* This paper is a modified version of Chapter III of my Ph.D. thesis (Université de Montréal, 1979). It was written while I was visiting the University of Pennsylvania. I would like to thank my supervisor, Marcel Boyer, the members of the dissertation's committee, Camille Bronsard, Richard Kihlstrom, and Claude Montmarquette, and my colleague François Vaillancourt for their comments. I remain responsible for any errors or shortcomings. The Société Saint-Jean-Baptiste (Prêt d'Honneur) and the Ministère des Affaires Sociales du Québec (C.Q.R.S.) gave financial support for this study.

1 In other words, an expert would not have purchased this amount of unnecessary services.

2 There are some exceptions; see Evans [8], [9], Frech III and Ginsburg [11], Fuchs [12]. 
study, however, the possibility that some suppliers may be motivated to oversell services to their clients when there is excess capacity will be considered ${ }^{3}$. The conditions under which an increase in insurance coverage for the services initially increases the real or effective cost of information and then increases the possibilities of fraud will be presented.

In this model, the behaviour of the consumer on the market is characterized by a decision rule which consists of accepting or declining a service offered, and returning to or avoiding the producer in the future. The decision of the consumer is based on his or her a priori information concerning the cost of the service. The consumer doesn't use market information to make his final decision in this model. But this possibility may be considered by using a Bayesian model of search (Dionne [4]).

The insurance policies studied are those of full insurance and of coinsurance. Experience rating is also analysed. The insurance policies may be complete or incomplete. By a full insurance policy one means that all the direct expenses are covered when the event, accident or illness, occurs.

An insurance policy may include a coinsurance clause. In this case the insurance company pays a fraction $(0<\alpha<1)$ of the loss when the event occurs, and the insured pays the balance, i.e. $(1-\alpha)$ of the amount of loss. An insurance policy is complete when all the costs of search have the same insurance coverage than the amount of loss, even if the consumer refuses the service. It is incomplete when the search costs are less covered than the service costs. Finally, experience rating is defined as the variation of the insurance premium in relation to the insured past experience of loss.

In the first section of the paper, the model of the producer under insurance is presented, followed by the analysis of the effect of insurance coverage on the behaviour of the consumer. In conclusion, the main results of the paper are summarized and an avenue of research is proposed.

\section{The producer}

In this kind of market, the service is offered jointly with a diagnosis (or an estimate). The consumer accepts or refuses the offer on the basis of the diagnosis since the service itself cannot be evaluated ex-ante. The price of the service includes a fixed cost for the diagnosis and the consumer must pay this fixed cost even if the offer is refused.

Since the technical information is distributed in an asymmetrical fashion in favor of the producer, the latter may be motivated to offer unnecessary services to the consumer. The object of this section is to present the effect of insurance on the optimal solution of the producer. Following Darby and Karni, let's assume that the producer faces a stochastic demand for its services and that there exists excess capacity in the market in the short run i.e. the waiting period for consumers services is null. Since it is difficult to accumulate stocks in a firm producing services, the producer may be

${ }^{3}$ For an analysis of the possibilities of unnecessary surgeries see McCarthy and Widmer [17], and Dionne [4]. 
motivated to offer more services to a non-perfectly informed consumer than as in a period of full capacity ${ }^{4}$. The result of this behavior is stochastic since the producer doesn't know whether or not the consumer will accept the supplementary amount of services. Moreover if the services are refused the consumer may go elsewhere immediately and the producer may lose present and future sales.

The amount of fraud is at its optimal level when the marginal expected utility of the producer is null. Let's assume the following expected utility in which the producer controls the total amount of services $(v \geqslant 0)$ for a given insurance coverage $(q \geqslant 0)$. The expected utility is equal to the utility of the profit corresponding to the present offer, times the probability that it will be accepted, plus the utility of the present value from future sales to the consumer, times the probability that the consumer will return in the future :

$$
E V=(1-F(v, q)) V(\ell(v)-C(v))+(1-P(v, q)) V(I)
$$

Where $E V$ is the expected utility ; $V^{\prime}>0$, and $V^{\prime \prime} \leqslant 0$;

$v \quad$ is the total amount of services prescribed ;

$q$ is the consumer's level of insurance coverage ;

$\ell(v)$ is the total revenue ${ }^{5} ; \ell_{v}>0, \ell_{v v}=0$ where $\ell_{v}=\frac{\partial \ell}{\partial v}$

$C(v)$ is the total cost ; $C_{v}>0, C_{v v}>0$;

$I \quad$ is the anticipated present value of future profits from a consumer ; $I$ is known and is contingent upon the consumer returning (there is no uncertainty on $I$ itself);

$F \quad$ is the estimation by the producer of the cumulative probability function that the consumer will refuse the present service ; $F_{v}>0$;

$P \quad$ is the estimation by the producer of the cumulative probability function that the consumer will refuse future services; $P_{v}>0$.

$F$ and $P$ are also function of $q$ for a given $\nu . F_{q}$ and $P_{q}$ represent the effect of insurance on the estimated cumulative probability functions. For example, $F_{q}<0$ means that the producer estimates that if the consumer increases insurance coverage, the probability that the consumer will refuse the services offered will be less. The reverse is true for $F_{q}>0$.

The equation (1) may be rewritten as follows :

$$
E V=[1-F(v, q)] V_{1}+[1-P(v, q)] V_{2}
$$

where $V_{1} \equiv V(\ell(v)-C(v))$;

$V_{2} \equiv V(I)$.

The optimal amount of $v$ for a given $q$ is given by:

$$
[1-F(v, q)] V_{1}^{\prime}\left(\ell_{v}-C_{v}\right)=F_{v} V_{1}+P_{v} V_{2}
$$

${ }^{4}$ The producer may also sell the service at a higher price. In Appendix 1, the model of the producer is generalized in order to take into account this possibility.

${ }^{5} \ell(v)$ is a two-part tariff consisting of a fixed cost of producing a consumer estimate $(\ell(0))$ and a linear price for services provided. 
In the first order condition ${ }^{6}$ the marginal benefit, in terms of utility associated with fraudulent services, is equal to the marginal cost, in terms of increasing the probabilities of losing the value of utilities associated to the amounts of present and future sales. For a given $q$, in order for fraud to occur, the marginal benefit in terms of utility of selling additional services to an expert buyer, must be greater than the marginal cost in terms of increasing the probabilities. This condition can easily be met when there are significant costs of diagnosis since the non expert buyers are not enough informed.

The effect of insurance on the optimal $v$ is obtained by differentiating (2) :

$$
\left.\frac{d v}{d q}\right|_{d I=0}=\frac{F_{q} V_{1}^{\prime}\left(\ell_{v}-C_{v}\right)+F_{v q} V_{1}+P_{v q} V_{2}}{\Delta}
$$

The sign of $\frac{d v}{d q}$ depends on the sign of the numerator. For example, if $F_{q}<0$ and if $P_{v q}, F_{v q} \leqslant 0$, then $\frac{d v}{d q}>0$. This means that the possibilities of fraud increase with insurance coverage. But many combinations are possible, and in order to obtain the true sign of $\frac{d v}{d q}$, the estimation of the functions $F(v, q)$ and $P(v, q)$ must be studied in detail. The behavior of the consumer in this kind of market will therefore be presented.

\section{The consumer}

\subsection{Risk averse consumer without insurance}

This model explains how the consumer decides to accept or decline a service offer and whether or not to return to the producer (garage owner, surgeon or doctor) in the future. This model is not a search model like those proposed by Stigler [21] and McCall [15], [16] in which the consumer decides to find the lowest price or cost before buying a good or service. Here the consumer searches by necessity since fraud is suspected. There is no optimal rule for the decision related to a reservation price or cost. Instead, the consumer makes the decision to accept or decline the service offer using a priori information.

There are two other differences to justify the use of this kind of model in those markets : the consumer doesn't necessarily know precisely the level of service needed for a given event and, more importantly, the consumer may be defrauded by a producer who wants to exploit this situation of asymmetrical information.

\footnotetext{
${ }^{6}$ The second order condition is equal to: $[1-F(v, q)] V_{1}^{\prime \prime}\left(\ell_{v}-C_{v}\right)^{2}$ $+[1-F(v, q)] V_{1}^{\prime}\left(-C_{v v}\right)-2 F_{v} V_{1}^{\prime}\left(\ell_{v}-C_{v}\right)-F_{v v} V_{1}-P_{v v} V_{2}=\Delta<0$. If $F(v, q)$ and $P(v, q)$ are cumulative normal functions, then $F_{v v}, P_{v v}>0$ when $F(v, q), P(v, q)<.5$.
} 
Before going to the market, the consumer doesn't know the distribution of the costs 7 in the market and, generally, doesn't know the true cost corresponding to the event. But the consumer may evaluate the cost from past experience or from the a priori information. This level of information is used to estimate the expected utility of the consumer.

There are two kinds of uncertainty in this model : the first type is similar to the usual uncertainty associated with search activity i.e. the consumer must compare an offer that is known with certainty $\left(\ell(\bar{v})_{i}\right)$ for example, with an uncertain prospect associated with one more visit and with a certain search cost $\ell(0)$. The other kind of uncertainty is related to the dispersion of the costs in the market. The consumer doesn't know with certainty if an observed high $\ell(\bar{v})_{i}$ is due to high costs of production, to fraud, or to a bad a priori evaluation of the true costs. The consumer will decide to accept or to decline a service offer by using his a priori expected utility as reference ${ }^{8}$ :

$$
E U(S)=\int_{0}^{R} U(\bar{S}-\ell(v)) H(\ell(v)) d \ell(v)
$$

where $E U(S)$ is the expected utility of the consumer measured from the a priori distribution of the costs of the service ;

$\bar{S} \quad$ is the initial wealth ;

$R \quad$ is the upper limit of $\ell(v) ; 0 \leqslant R \leqslant \infty$. In the case of an automobile accident $R$ may be interpreted as the replacement cost of the car; for illness the interpretation of $R$ is more difficult but it will be assumed that the consumer knows the value of $R$;

$\ell(v) \quad$ is the total cost of purchasing a diagnosis or estimate and $v$ units of the service ;

$\ell(0) \quad$ is the fixed cost associated with a diagnosis or an estimate ;

$H \quad$ is an a priori probability density function; in the text $H(\cdot)$ will designate $H(\ell(v))$.

A decision-maker is risk averse if the utility function of wealth is concave, this is, $U^{\prime \prime}(S)<0$. Moreover Arrow [1] and Pratt [20] have presented two measures of risk aversion (absolute and relative), since the utility function is unique to a linear transformation. Arrow has also suggested two assumptions on the variation of the two measures :

$H_{1}$ : absolute risk aversion decreases as wealth increases; writing $r_{U}$ for absolute risk aversion, $r_{u}(S)=-\frac{U^{\prime \prime}(S)}{U^{\prime}(S)}$ and $\frac{d r_{U}}{d S}<0$

$\mathrm{H}_{2}$ : relative risk aversion increases as wealth increases; writing $S r_{U}$ for relative risk aversion, $S r_{U}=-\frac{S U^{\prime \prime}(S)}{U^{\prime}(S)}$ and $\frac{d\left(S r_{U}\right)}{d S}>0$.

7 The term cost is used in a general sence : different costs may represent different prices for a given quantity of services or different quantities of services for a given event. By assumption, the quality of services should remain constant from one agent to another.

${ }^{8}$ It is assumed implicitly that the consumer will not reevaluate the a priori cost distribution during the course of the search process. 
A consumer with utility function $U_{1}$ is more risk averse than another one with utility function $U_{2}$ if $r_{U_{1}}>r_{U_{2}}$ for all $S$. Finally Pratt has defined a risk premium as the amount $(\pi>0)$ which makes the consumer indifferent between receiving a random amount $S$ and receiving a certain amount $E(S)-\pi(S)$ :

$$
E U(S)=U(E(S)-\pi(S))
$$

Now let's suppose that the $i^{\text {th }}$ producer prescribes a level of services associated with the cost $\ell(\bar{v})_{i}$. The consumer may accept or decline the offer. If the consumer accepts, the total cost in utility terms will be $U\left(\bar{S}-\ell(\bar{v})_{i}\right)$; if the consumer declines, and goes elsewhere the fixed cost associated with the diagnosis or the estimate must be paid. In the later case the new expected utility will be $E U(S-\ell(0))$.

Following Darby and Karni, the decision rule is :

a) if $U\left(\bar{S}-\ell(\bar{v})_{i}\right) \geqslant E U(S)$; the consumer accepts the service offer since the certain utility associated with it is greater than the a priori expected utility. The consumer does not suspect fraud and will therefore come back in the future should the event reoccur;

b) if $E U(S-\ell(0)) \leqslant U\left(\bar{S}-\ell(\bar{v})_{i}\right)<E U(S)$; the consumer suspects fraud but accepts the level of service since the expected utility to go elsewhere is less or equal than the certain utility associated with the service offer. But, in this case, the consumer will not come back in the future;

c) if $E U(S-\ell(0))>U\left(\bar{S}-\ell(\bar{v})_{i}\right)$; the consumer declines the offer and goes elsewhere since : 1) fraud is suspected and 2) the expected utility to go elsewhere is greater than the certain utility associated with $\ell(\bar{v})_{i}$. Of course, the consumer will not come back in the future.

The graphical representation of this decision rule for an individual who is risk averse is :

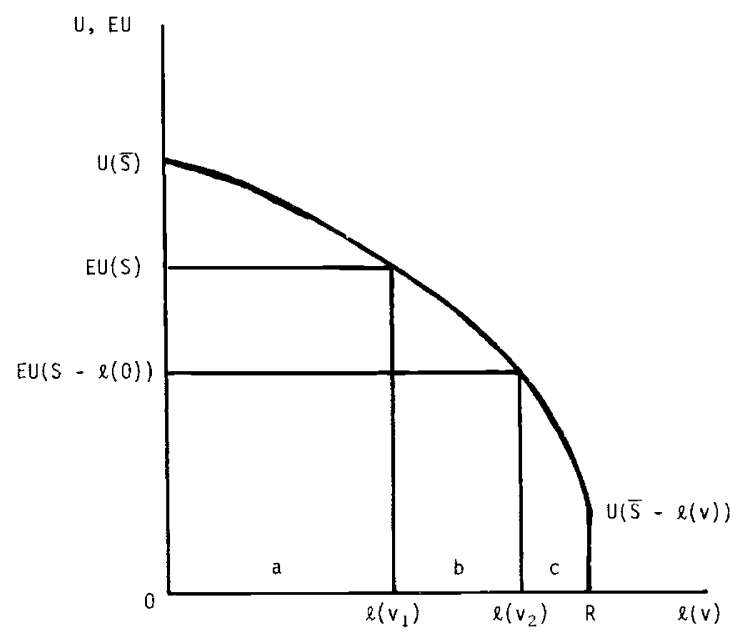

Figure 1 
The limits of regions $a, b, c$ are obtained when ${ }^{9}$ :

$$
U\left(\bar{S}-\ell\left(v_{1}\right)\right)=E U(S) \Longrightarrow \ell\left(v_{1}\right)=E \ell(v)+\frac{\sigma^{2}}{2} r_{U}(\bar{S})
$$

where $\sigma^{2} \quad$ is the variance of the distribution ;

$r_{u} \quad$ is defined as before; and

$\frac{\sigma^{2}}{2} r_{U}$ is the risk premium.

$$
\begin{aligned}
& U\left(\bar{S}-\ell\left(v_{2}\right)\right)=E U(S-\ell(0)) \Longrightarrow \ell\left(v_{2}\right)=E \ell(v)+\ell(0)+\frac{\sigma^{2}}{2} r_{U}(\bar{S}-\ell(0)) \\
& U(\bar{S}-\ell(v))=U(S-R)
\end{aligned}
$$

The producer evaluates regions $a$ and $b$ respectively when estimating the values of $P(v, q)$ and $F(v, q)$ for a given consumer.

\subsection{Risk averse consumer with insurance}

The a priori expected utility of the consumer insured against the loss associated with an accident or illness may be written as follows

$$
E U(S, \alpha, k)=\int_{0}^{R} U(\bar{S}-\ell(v)+q) H(\cdot) d \ell(v)
$$

where $q=\alpha(\ell(v)-k)$

$$
\begin{aligned}
& k \geqslant 0 \\
& 0 \leqslant \alpha \leqslant 1 ;
\end{aligned}
$$

$q$ is the insurance benefit received by the insured; $q=0$ if $\ell(v)<k$;

$\alpha$ is the percent of the loss paid by the insurer;

$k$ is a deductible amount $; k \geqslant 0$.

Let's assume that the insurance premium $(P(\alpha, k))$ is null since there is no choice of insurance policy in the model ${ }^{10}$. Therefore this model can also apply for cumpulsory insurance. Here we want to show how the variation of insurance coverage can affect the consumer's search behavior when the event has occurred.

From this general formulation different particular cases may be obtained :

$$
\begin{array}{ll}
\text { No insurance } & : k=0, \alpha=0 \\
\text { Coinsurance without deductible } & : k=0,0<\alpha<1 \\
\text { Full insurance without deductible : } k=0, \alpha=1 \\
\text { Full insurance with deductible } & : k>0, \alpha=1 \\
\text { Coinsurance with deductible } & : k>0,0<\alpha<1
\end{array}
$$

To measure the effect of a change in insurance coverage on the producer's possibilities of fraud, one must calculate the variation of the regions $a, b$, and $c$ when $q$ varies. If for example region $a$ increases, this means that the region in which the consumer doesn't suspect any kind of fraud increases and therefore the possibilities of

9 This result is obtained by using Taylor's series approximation to expand the utility function of wealth around both sides of (4.1). See Pratt [20].

10 Dionne [5] presented a model of search with the choice of insurance policy. 
fraud increase for the producer ; if region $a$ doesn't change, the possibilities of fraud do not change, and if region $a$ decreases, the possibilities of fraud decrease, ceteris paribus.

The same analysis can be applied to region $b$. But the interpretation of the changes is not the same for both regions : for region $b$, the variation of the possibilities of fraud concerns the levels of services that are accepted, since the cost of search is too high ; these possibilities will be called short run possibilities of fraud. For region a, long run possibilities of fraud are considered since the consumer is concerned with the possibility to come back in future. For region $c$, with a constant $R$, its variation is due to the variation of regions $a$ and $b$.

The insurance benefits $(q)$ may vary in two ways : by a variation of $\alpha$ or by a variation of $k$. In this paper the analysis shall limit itself to the variation of $\alpha^{11}$.

Definition I (Dionne [6]) :

An insurance policy is complete when the search costs have the same insurance coverage as the costs associated with an event ${ }^{12}$. It is incomplete when the search costs are less covered than the service costs.

For example, if all the costs of diagnosis and consultation have the same insurance coverage as the costs of surgery, then insurance policy is complete if there are no other search costs.

\section{Definition II :}

The real or effective cost of search corresponding to an insurance policy, is defined as the cost of search paid by the consumer under that insurance policy, weighted by the insurance coverage of the costs of the services.

For example, under an insurance policy characterized by 1) a coinsurance rate $\alpha$ $(0<\alpha<1), 2)$ a null deductible and, 3) a complete coverage of the direct search costs, $\ell(0)$ is the cost of the diagnosis, $d=\ell(0)(1-\alpha)$ is the cost or search paid by the consumer and $\delta=\frac{d}{(1-\alpha)}=\ell(0)$ is the real cost of search. Here the real cost of search is equal to the cost of search. However when the insurance policy is incomplete, $d=\ell(0)$ and $\delta=\frac{\ell(0)}{(1-\alpha)}>\ell(0)$. Since the cost of search is not covered by insurance, the real cost of search faced by the consumer is greater than the cost of search. The weight of the insurance coverage permits to take account of how the cost of search is covered by the insurance policy and, as it will be showed in the following propositions, permits to compare the anticipated benefits of search to the real cost of search faced by the consumer under insurance. Moreover when $\alpha=1$, under an incomplete insurance policy $\delta=\infty$ and under a complete insurance policy $\delta$ is undetermined. In other words, under full insurance, the consumer faces an infinite real cost of search and will not search when the insurance policy is incomplete and is indifferent when the insurance policy is complete since both the cost and the benefits of search are null. For other examples, see Appendix 2.

11 Many extensions of the paper concerning the variation of the deductible are available upon request from the author.

12 This definition is more general than that proposed by Dionne [5]. Here all the search costs are covered. In the text, " real" and "effective" have the same meaning. 


\section{Proposition I:}

If the utility function is concave (risk averse) and if there is no indirect cost of search ${ }^{13}$, the possibilities of fraud decrease with respect to an increase in the coinsurance rate in policy characterized by : a) a null deductible, b) a complete coverage of the direct search costs.

The proof of this proposition is obtained by differentiating the frondiers of regions $a$ and $b$ with respect to $\alpha$. At $\ell) v_{1}$ ) we have :

$$
U\left(\bar{S}-(1-\alpha) \ell\left(v_{1}\right)\right)=E U(\bar{S}-(1-\alpha) \ell(v))
$$

which implies that

$$
\begin{aligned}
& \ell\left(v_{1}\right)=\mathrm{E} \ell(v)+(1-\alpha) \sigma^{2} / 2 r_{U}(\bar{S}) \\
& \frac{d \ell\left(v_{1}\right)}{d \alpha}=-\frac{\sigma^{2}}{2} r_{U}<0 .
\end{aligned}
$$

and

At $\ell\left(v_{2}\right)$, under a complete insurance policy :

$$
U\left(\bar{S}-(1-\alpha) \ell\left(v_{2}\right)\right)=E U(\bar{S}-(1-\alpha)(\ell(v)+\ell(0)))
$$

which implies that

$$
\ell\left(v_{2}\right)=\mathrm{E} \ell(v)+\ell(0)+(1-\alpha) \sigma^{2} / 2 r_{U}(\bar{S}-\ell(0))
$$

and $\quad \frac{d \ell\left(v_{2}\right)}{d \alpha}=-\frac{\sigma^{2}}{2} r_{v}<0$

$\ell\left(v_{1}\right)$ and $\ell\left(v_{2}\right)$ decrease with an increase of $\alpha$. This means that search increases with insurance coverage since the risk premium decreases with an increase of $\alpha$. In other words, as $\alpha$ increases, the risk associated to search activity decreases. This result is similar to that obtained by Dionne [5] and is explained by the fact that the partial risk aversion is increasing in wealth. The partial risk aversion is measured by:

$Z(S, W)=-S \frac{U^{\prime \prime}(S+W)}{U^{\prime}(S+W)}$.

According to Diamond and Stiglitz [3, p. 356], the partial risk aversion is increasing in wealth if this following sufficient condition is fulfilled: $\frac{d r_{U}(S+W)}{d(S+W)}<0$ and $\frac{d\left((S+W) r_{U}\right)}{d(S+W)}>0$.

In this case, if the insurance coverage increases, the risk premium decreases more than proportionaly to the variation of the insurance coverage that is :

$$
\frac{\partial}{\partial \alpha}\left(\frac{\pi(\bar{S},(1-\alpha) \ell(v))}{(1-\alpha)}\right)<0 \text { as } \frac{\partial Z(S, W)}{\partial S}>0
$$

where $\pi(\bar{S},(1-\alpha) \ell(v)) \equiv(1-\alpha)^{2} \frac{\sigma^{2}}{2} r_{U}(\bar{S})$ at $\ell\left(v_{1}\right)$. The same analysis applies at $\ell\left(v_{2}\right)$.

${ }_{13}$ This assumption will be dropped. Costs related to time and transportation are examples of indirect costs of search. 
Figure 2 shows a local decrease of the possibilities of fraud in both the short and the long run: the boundaries of regions $a$ and $b$ move to the left hand side as $\alpha$ increases. When $\alpha=1$, both (6) and (7) become

$$
U(\bar{S})=E U(\bar{S}) \quad \forall \ell(v)
$$

which implies that the frontiers of regions $a$ and $b$ are undetermined.

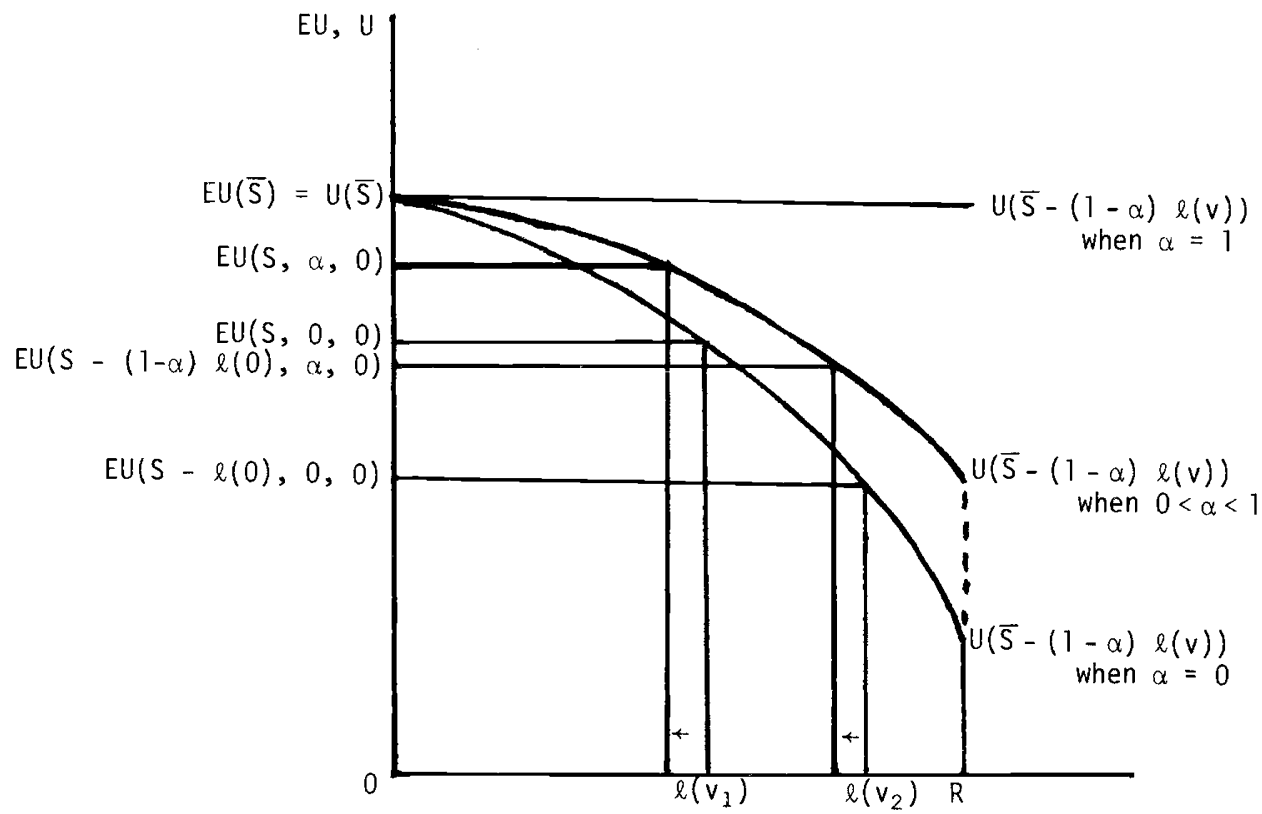

Figure 2

\section{Corollary I:}

If the utility function is linear (risk neutral) and if the same conditions as in Proposition $I$ are followed, the possibilities of fraud do not change with respect to an increase in the coinsurance rate.

The proof is straightforward since the risk premium is null and the real cost of search does not vary under a complete coverage of the direct seach costs.

\section{Proposition II :}

If the utility function is concave (risk averse) and if there is no indirect cost of search, the possibilities of fraud in the short run may increase or decrease with respect to an increase in the coinsurance rate in an insurance policy characterized by a) a null deductible, b) an incomplete coverage of the direct search costs. 
In the proof we assume that all the search costs are not covered by insurance in the case of an incomplete insurance policy. But the results may apply to all situations where the average insurance coverage of the search costs is less than the insurance coverage of the service costs.

The analysis for region $a$ is the same as in Proposition $I$. Now at $\ell\left(v_{2}\right)$ :

$$
U\left(\bar{S}-(1-\alpha) \ell\left(v_{2}\right)\right)=E U(\bar{S}-(1-\alpha) \ell(v)-\ell(0))
$$

which implies that

and

$$
\ell\left(v_{2}\right)=E \ell(v)+\frac{\ell(0)}{(1-\alpha)}+(1-\alpha) \sigma^{2} / 2 r_{l}(\bar{S}-\ell(0))
$$

$$
\frac{d \ell\left(v_{2}\right)}{d \alpha}=\frac{\ell(0)}{(1-\alpha)^{2}}-\frac{\sigma^{2}}{2} r_{v} \gtreqless 0
$$

as

$$
\frac{\ell(0)}{(1-\alpha)^{2}} \gtreqless \frac{\sigma^{2}}{2} r_{l^{\prime}} .
$$

Here there is a trade-off between a decrease in the risk premium and an increase in the real cost of search. But this is a local trade-off, and differentiating (8) a second time yields :

$$
\frac{d^{2} \ell\left(v_{2}\right)}{d \alpha^{2}}=2 \frac{\ell(0)}{(1-\alpha)^{3}}>0
$$

As $\alpha \rightarrow 1$, the effect of the increase in the real cost of search becomes more important than the decrease in the risk premium and, then, the possibilities of fraud increase. This means that more consumers will accept fraudulent services in the short run since the real cost of search increases with the increase of insurance coverage faster than the risk premium decreases. Therefore, empirical studies would probably find that the possibilities of fraud increase with respect to an increase in the coinsurance rate, even if the theoretical sign is locally ambiguous. The article in the New York Times, mentioned in the introduction of the paper, seems to confirm this prediction. Finally for many consumers there are no long run possibilities of fraud in this kind of market, since a particular operation or automobile accident may never reoccur. Figure 3 shows an increase of the possibilities of fraud in the short run: the boundary of region $b$ goes from $\ell\left(v_{2}\right)$ to $\ell\left(v_{2}\right)^{\prime}$ as $\alpha$ increases.

\section{Corollary $I I$ :}

If the utility function is linear (risk neutral) and if the same conditions as in Proposition $I I$ are followed, the possibilities of fraud in the short run increase with respect to an increase in the coinsurance rate.

The proof is straightforward since the risk premium is null and the real cost of search increases under an incomplete coverage of the direct search costs.

Moreover in Figure 3, if $\alpha=1$, the region $a$ becomes OR since $U(\bar{S})>E U(\bar{S}-\ell(0)) \forall \ell(v)$. Under full insurance the consumer is indifferent to any level of service and is not concerned with fraud since the marginal cost of the service is null and the real cost of search approaches infinity as $\alpha$ approaches one. 


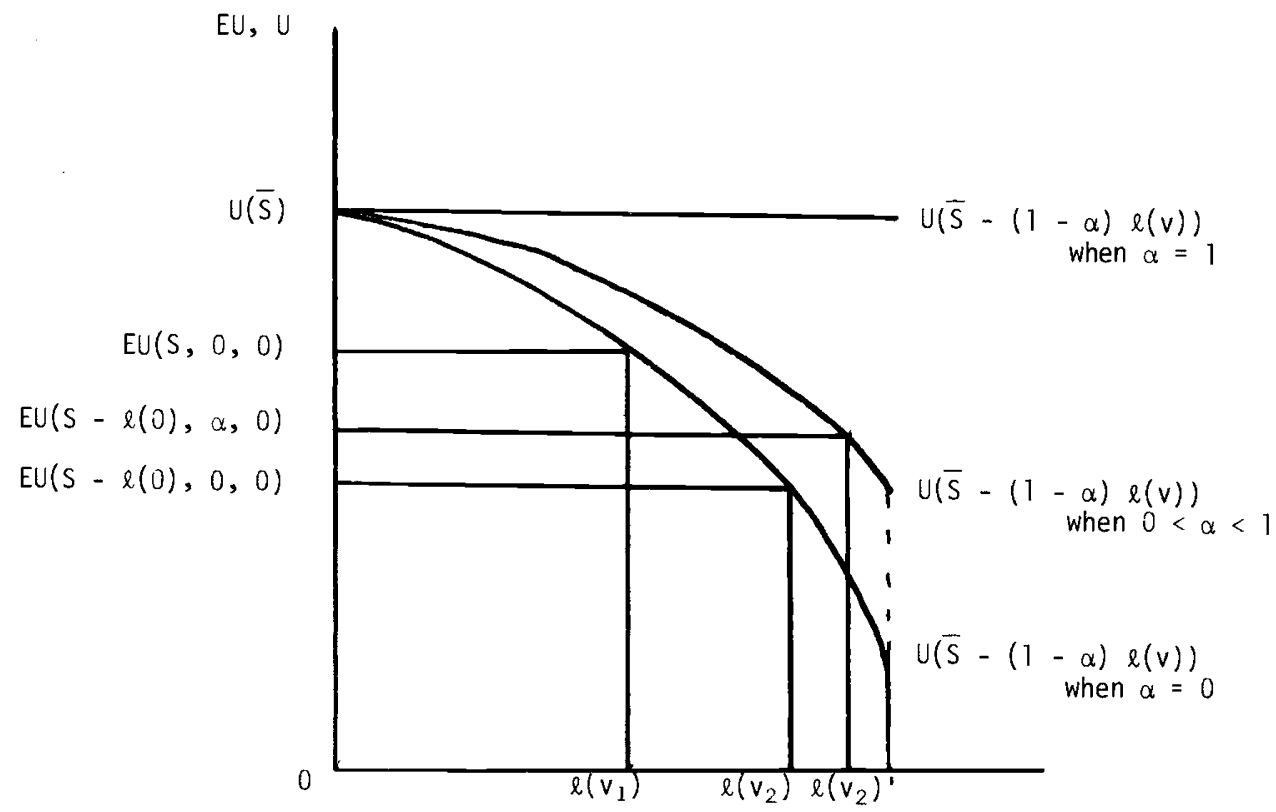

Figure 3

\subsection{The price of time ${ }^{14}$ and the possibilities of fraud}

In this section it will be assumed that the time used to search is working time. But it would be easy to generalize the model and to introduce leisure time. We can introduce the price of time by redefining $\bar{S}$ as :

$$
\bar{S}=W T
$$

where $W$ is the wage bill per unit of time;

$T$ is the total working time for a given period.

$T$ may be defined as a function of search time :

$$
T=T_{0}-t x
$$

where $T_{0}$ is constant ;

$t$ is the total time used for a diagnosis or an estimate ;

$x$ is the number of diagnoses or estimates.

Therefore the a priori expected utility of the consumer may be rewritten as follows $(x=1)$ :

$$
E U\left(W\left(T_{0}-t\right), \alpha, 0\right)=\int_{0}^{R} U\left(W\left(T_{0}-t\right)-(1-\alpha) \ell(v)\right) H(\cdot) d \ell(v)
$$

14 The introduction of other indirect costs of search such as transportation costs can be done in a similar way. 


\section{Proposition 111 :}

If $U$ is concave and if there are indirect costs of search, the possibilities of fraud in the short run may increase or decrease with respect to an increase in the coinsurance rate in an insurance policy characterized by: a) a null deductible, b) a complete coverage of the direct search costs.

The proof is similar to the proof of Proposition II. The possibilities of fraud may increase even under a regime of complete coverage of the direct search costs, since the real time cost of search increases with $\alpha$.

\subsection{Experience rating and the possibilities of fraud}

Experience rating is defined as a modification of the rate of insurance to reflect the insured's past experience. Since experience rating modifies insurance rates, we will modify the expected utility in the following way: the insured now considers the effect of his or her present behavior on the insurance premium. Therefore the expected utility becomes :

$$
E U(S, \alpha, 0)=\int_{0}^{R} U(\bar{S}-\ell(v)+q-B(q)) H(\cdot) d \ell(v)
$$

where $q=\alpha \ell(v)$

$0 \leqslant \alpha \leqslant 1$

$B(q)$ is additional payment at the end of the year depending on the loss experience during the year. ${ }^{15}$

The function $B(q)$ may be specified by using a modification factor reflecting past experience :

$$
M(q)=\frac{(q-u)}{u} \cdot \lambda
$$

where $M(q)$ is the percentage by which the pure premium is altered ;

$u \quad$ is the pure premium reflecting the anticipated loss of the insured;

$q \quad$ is the insured's actual loss experience covered by the insurance ;

$\lambda \quad$ is the credibility factor which measures the statistical significance of the insured's loss experience, $0 \leqslant \lambda \leqslant 1$.

If we assume that the total premium is limited to the pure premium, $B(q)$ is equal to:

$$
B(q)=M(q) \cdot u \equiv \lambda(q-u) \gtreqless 0
$$

as

$$
q \gtreqless u \text { and } b_{q}=\lambda \geqslant 0 .
$$

\section{Example I:}

If $U$ is concave, if there is no indirect cost of search and if the insurance premium is a function of the past experience of the insured (as assumed above) the variation of the possibilities of fraud with respect to $\alpha$ in the short run is less important under

15 The same results can be obtained in a two periods model if the insured considers the effect of his or her present behavior on the future insurance premium. 
experience rating than without experience rating in an insurance policy characterized by : a) null deductible, b) an incomplete coverage of the direct search costs.

At $\ell\left(v_{2}\right)$ we have under incomplete insurance coverage :

$$
\ell\left(v_{2}\right)=E \ell(v)+\frac{\ell(0)}{(1-\alpha+\alpha \lambda)}+(1-\alpha+\alpha \lambda) \sigma^{2} / 2 r_{U}(\bar{S}-\ell(0))
$$

and

$$
\begin{aligned}
\frac{d \ell\left(v_{2}\right)}{d \alpha} & =\frac{\ell(0)(1-\lambda)}{(1-\alpha+\alpha \lambda)^{2}}-(1-\lambda) \sigma^{2} / 2 r_{U} \\
& =(1-\lambda)\left[\frac{\ell(0)}{(1-\alpha+\alpha \lambda)^{2}}-\sigma^{2} / 2 r_{U}\right]<\frac{\ell(0)}{(1-\alpha)^{2}}-\sigma^{2} / 2 r_{L^{\prime}}
\end{aligned}
$$

- if $\lambda=0$, this corresponds to no experience rating, therefore the result is the same as in Proposition $I I$;

- if $\lambda=1$, this corresponds to no insurance ;

- if $0<\lambda<1$ and if $r_{U}=0$, the insurance premium is function of past experience and the possibilities of fraud are less important than in the case without experience rating since experience rating reduces the variation of the real cost of search ;

- if $0<\lambda<1$, and under risk aversion, the possibilities of fraud are still less important than in the case without experience rating since the reduction of the variation of the risk premium is less important than the reduction of the variation of the real cost of search for all $0<\alpha<1$.

This result applies also in the case of full insurance and the limits of regions $a$ and $b$ may now be inferior to $R$ under full insurance and experience rating. But this kind of insurance policy is restricted to clients who have a sample large enough in order that $\lambda \neq 0$, such as for group health insurance. In that particular case the individual incentive created by experience rating will be function of the rating rule in the group.

\section{Summary of results and conclusion}

In this conclusion we summarize the results presented in the section on the consumer and link those results with the possibilities of fraud by the producer. We known that the producer can evaluate subjectively regions $a, b, c$, by estimating the probabilities $F(v, q)$ and $P(v, q)$. Moreover, the sign of $\frac{d v}{d q}$ depends on the sign of the variation of $F_{v}, F_{r q}$ and $P_{v q} \cdot P(v, q)$ is the cumulative probability function, estimated by the producer, that the consumer will refuse future services. It is associated with region $a$. $P_{v q}$ measures the effect of insurance on $P_{v}$. If $P_{v q}<0$, an increase in insurance coverage reduces the marginal effect of the increase in the amount of services on probability that the consumer will refuse future services (long run). In other words, $P_{v q}$ will be $<0$ if region $a$ increases. 
$F(v, q)$ is the cumulative probability function, estimated by the producer, that the consumer will refuse the present service (short run). It is associated with region $b . F_{v}$ and $F_{v q}$ will be $<0$ if region $b$ increases.

The presentation of the following results assumes implicitly that the producer is aware of consumer's insurance policy. The cost of this information is assumed null, since the information is directly obtained from the consumer. If the consumer doesn't collaborate, this lack of information may be treated as a supplementary source of uncertainty for the producer ${ }^{16}$.

Different results on the variation of the amount of coinsurance are obtained under the hypotheses of risk aversion and risk neutrality for both complete and incomplete insurance policies. In a complete insurance policy, the possibilities of fraud do not change (Corollary I) with the increase of the coinsurance rate under the hypothesis of risk neutrality, but decrease (Proposition I) under risk aversion. In the case of an incomplete insurance policy with risk neutrality, the possibilities of fraud increase along with the coinsurance rate (Corollary II), but may increase or decrease under risk aversion (Proposition II). Risk aversion eliminates units results here. There is a trade-off between a decrease in the risk premium and an increase in the real cost of search. This, however, is a local trade-off and, as mentioned in the text, empirical studies would probably find that the possibilities of fraud increase with respect to an increase in the coinsurance rate, even if the theoretical sign is locally ambiguous.

Experience rating reduces the possibilities of fraud but doesn't eliminate it when the credibility factor is inferior to one (Example I). One other extension of the basic model has been presented. The introduction of the price of time increases the possibilities of fraud since it increases the real cost of search, but it also accentuates the effect of the variation of the coinsurance rate (Proposition III).

In addition to these results, it has also been demonstrated that, under full and incomplete insurance, the possibilities of fraud increase considerably since the marginal cost of the service to the consumer is null. This result is true regardless of consumer risk behavior.

This analysis of the effects of insurance on the possibilities of fraud does not take into account the administrative measures used by the insurers to limit fraud. For example, in automobile collision insurance, insurers may give the name and address of a qualified and honest repair service to the insured or they may use an evaluation to estimate the cost of an accident and negociate with a garage owner the cost of the repair. In medical care markets certain insurers have their own surgeons i.e. group practice, but others ask their clients to obtain a second opinion before proceeding with surgery. These measures do not penalize consumers due to producers' fraud but they do increase the insurance premiums. One avenue of research would be to compare these administrative measures with partial insurance coverage and experience rating in order to determine the best way in which to reduce fraud.

16 Often, in practice, insurance policies do not vary for a given event and the producer needs only to know whether or not the consumer is insured. 


\section{Appendix 1}

The generalization of the producer's model

Under the market conditions described in section I of this paper, a producer may be motivated to offer more services to a consumer than in a situation of full capacity or to sell the services at higher prices. These behaviors may be represented in maximizing the relation (Al) with respect to $v$ and $p$ where $p$ is the price of one unit of $v$ :

(Al)

$$
\underset{v, p}{\operatorname{Max}} E V=[1-F(v, p, q)] V(p \cdot v-C(v))+[1-P(v, p, q)] V(I) .
$$

In defining $V_{1}=V(p \cdot v-C(v))$ and $V_{2}=V(I)$, (Al) may be rewritten :

$$
\operatorname{Max}_{v, p} E V=[1-F(v, p, q)] V_{1}+[1-P(v, p, q)] V_{2}
$$

The first order conditions give :

$$
\begin{aligned}
& \frac{\partial E V}{\partial v}=[1-F(v, p, q)] V_{1}^{\prime}\left(p-C_{v}\right)-F_{v} V_{1}-P_{v} V_{2}=0 \\
& \frac{\partial E V}{\partial v}=[1-F(v, p, q)] V_{1}^{\prime}(v)-F_{p} V_{1}-P_{p} V_{2}=0
\end{aligned}
$$

The second order conditions are equal to :

$$
\frac{\partial^{2} E V}{\partial v^{2}}=\Delta_{v v}<0
$$

when $V_{i}^{\prime \prime} \leqslant 0, F_{v v}>0$ and $P_{v v}>0$.

$$
\frac{\partial^{2} E V}{\partial p^{2}}=\Delta_{p p}<0
$$

when $V_{i}^{\prime \prime} \leqslant 0, F_{p p}>0$ and $P_{p p}>0$.

$$
\Delta_{v p}=\Delta_{p v}=0
$$

by hypothesis ; it is supposed that one behavior does not affect the marginal effect of the other one.

This is a maximum since :

$$
\Delta_{v v}<0 \text { and } \Delta=\left|\begin{array}{cc}
\Delta_{v v} & 0 \\
0 & \Delta_{p p}
\end{array}\right|>0
$$

The effects of insurance on these two behaviors are given by :

$$
\begin{aligned}
& \Delta_{v v} d v+\Delta_{v p} d p=\left[F_{q} V_{1}^{\prime}\left(p-C_{v}\right)+F_{v q} V_{1}+P_{v q} V_{2}\right] d q \\
& \Delta_{p v} d v+\Delta_{p p} d p=\left[F_{q} V_{1}^{\prime}(v)+F_{p q} V_{1}+P_{p q} V_{2}\right] d q
\end{aligned}
$$

And using the Cramer's rule

$$
\begin{aligned}
& \frac{d v}{d q}=\frac{\Delta_{p p}}{\Delta}\left[F_{q} V_{1}^{\prime}\left(p-C_{v}\right)+F_{v q} V_{1}+P_{v q} V_{2}\right] \gtreqless 0 \\
& \frac{d p}{d q}=\frac{\Delta_{v v}}{\Delta}\left[F_{q} V_{1}^{\prime}(v)+F_{p q} V_{1}+P_{p q} V_{2}\right] \gtreqless 0
\end{aligned}
$$

The sign of these variations depends of the signs of $F_{q}, F_{v q}, P_{v q}, F_{p q}$ and $P_{p q}$ which are explained in section 3 . 
Appendix 2

The real or effective cost of search

No insurance

Coinsurance with no deductible and complete coverage

$0<\alpha<1, k=0$

Coinsurance with no deductible and non complete coverage

$0<\alpha<1, k=0$

Full insurance with no deductible and complete coverage

$\alpha=1, k=0$

Coinsurance with positive deductible and non complete coverage $0<\alpha<1, k>0, \ell(0) \leqslant k$

Coinsurance with positive deductible and complete coverage $0<\alpha<1, k>0, \ell(0)>k$

$$
\begin{aligned}
& d=\ell(0)=\delta \\
& d=(1-\alpha) \ell(0) \quad, \quad \delta=\ell(0)
\end{aligned}
$$$$
d=\ell(0)
$$$$
\delta=\frac{\ell(0)}{(1-\alpha)}
$$

$$
d=0
$$

$\delta$ is undetermined

\section{REFERENCES}

1. ARROW, K. J.: "Uncertainty and the welfare economics of medical care", American Economic Review, 53 (December 1963), 941-973.

2. DARBY, M., and KARNI, E. : "Free competition and the optimal amount of fraud", Journal of Law and Economics, 14 (April 1973), 67-88.

3. DIAMOND, P. A., and STIGLITZ, J.E.: "Increases in risk and in risk aversion", Journal of Economic Theory, 8 (July 1974), 337-360.

4. DIONNE, G.: “Analyse des effets de l'assurance et de la relation de confiance consommateur-producteur sur les possibilités d'abus des chirurgiens", Actualité Economique, 56 (avril-juin 1980), 211-238.

5. DIONNE, G. : "Moral hazard and search activity", Journal of Risk and Insurance, 58 (September 1981), 422-435.

6. DIONNE, G.: "Search and insurance" International Economic Review (forthcoming).

7. DOHAN, M. R. : “ Cost maximisation and buyer dependence on seller provided information ", Journal of Economic Issues, 10 (No. 2, 1976), 430-452.

8. EVANS, R.G.: Price Formation in the Market for Physician Services in Canado 1957-1969, Information Canada, 1972. 
9. EVANS, R. G. : “ Models, markets and medical care”, in Issues in Canadian Economics, Officer and Smith (Ed.), McGraw-Hill Ryerson Limited, 1974, 197-206.

10. FELDSTEIN, M.S.: "Econometric studies of health economics", in Frontiers of Quantitative Economics, Intriligator and Keadack (Ed.), North-Holland Publications Co., 1974.

11. FRECH, III, H. E., and GINSBURG, P. B.: " Imposed health insurance in monopolistic market : a theoretical analysis", Economic Inquiry, 13 (March 1975), 55-70.

12. FUCHS, V.R.: "The supply of surgeons and the demand for operations", The Journal of Human Resources, 13 (supplement, 1978), 35-56.

13. HALL, J. R. : “Expected utility maximizing job search in a dynamic economic system", Unpublished paper, UCLA, 1975.

14. HALL, J. R., LIPPMAN, S. A., and McCALL, J. J.: " Expected utility maximization job search", in Studies in the Economics of Search, S. A. Lippman and J. J. McCall (Ed.), North-Holland, 1979, 133-156.

15. McCALL, J. J.: “The economics of information and optimal stopping rules" Journal of Business, 38 (July 1965), 300-317.

16. McCALL, J. J.: “Economics of information and job search", Quarterly Journal of Economics, 84 (February 1970), 113-126.

17. McCARTHY, E. G., and WIDMER, G. W. : "Effects of screening by consultants on recommended elective surgical procedures", New England Journal of Medecine (December 1974), 1331-1335.

18. NACHMAN, D. C.: “On risk aversion and optimal stopping”, Unpublished paper, Northwestern University, 1972.

19. PAULY, M. V.: "The economics of moral hazard", American Economic Review, 58 (June 1968), 531-537.

20. PRATT, J.: "Risk aversion in the small and in the large", Econometrica, 32 (JanuaryApril 1964), 122-136.

21. STIGLER, G.: “The economics of information", Journal of Political Economy, 69 (June 1961), 213-225.

22. ZECKHAUSER, R. :" Medical insurance: a case of study of the trade off between riskspreading and appropriate incentives", Journal of Economic Theory, 2 (March 1970), 10-26. 